\title{
Ongoing increasing temporal and geographical trends of the incidence of extended-spectrum beta-lactamase- producing Enterobacteriaceae infections in France, 2009 to 2013
}

\author{
I Arnaud ${ }^{1}$, S Maugat ${ }^{2}$, V Jarlier ${ }^{3}$, P Astagneau ${ }^{14}$, for the National Early Warning, Investigation and Surveillance of Healthcare- \\ Associated Infections Network (RAISIN)/multidrug resistance study group ${ }^{5}$ \\ 1. Regional Coordinating Centre for Healthcare-Associated Infections Control (CClin Paris - Nord), Paris, France \\ 2. French Institute for Public Health Surveillance (Institut de Veille Sanitaire, InVS), Saint Maurice, France \\ 3. AP-HP (Assistance Publique - Hôpitaux de Paris), Paris, France \\ 4. École des hautes études en santé publique (EHESP) Sorbonne Paris Cité University, Paris, France \\ 5. Members of the group are listed at the end of the article.
}

Correspondence: Isabelle Arnaud (isabelle.arnaud@aphp.fr)

Citation style for this article:

Arnaud I, Maugat S, Jarlier V, Astagneau P. Ongoing increasing temporal and geographical trends of the incidence of extended-spectrum beta-lactamase-producing Enterobacteriaceae infections in France, 2009 to 2013. Euro Surveill. 2015;20(36): pii=30014. DOI: http://dx.doi.org/10.2807/1560-7917.ES.2015.20.36.30014

Extended-spectrum beta-lactamase-producing Enterobacteriaceae (ESBL-E) are a major focus of multidrug-resistant organisms (MRO) surveillance programmes in France. To describe the temporal and geographical trends of these pathogens, we conducted an epidemiological study based on data extracted from the nationwide MRO surveillance network from 2009 to 2013. During this time, the incidence of ESBL-E infections in French hospitals increased by $73 \%$, from 0.35 to 0.60 per 1,000 patient days (PD) $(p<0.001)$ and ESBL-E bacteraemia by $77 \%$, from 0.03 to 0.05 per 1,000 PD ( $p<0.001)$. The incidence of ESBL-E infections was higher in intensive-care units (1.62 to 2.44 per 1,000 PD ( $p<0.001)$ ) than in recovery and long-term care facilities $(0.20$ to 0.31 per 1,000 PD $(p<0.001))$. Escherichia coli was the most frequent extended-spectrum betalactamase-producing (ESBL) pathogen, representing $59 \%(26,238 / 44,425)$ of all ESBL isolates, followed by Klebsiella pneumoniae $(20 \% ; 8,856 / 44,425)$ in 2013. The most frequent infection was urinary tract infection, for all species. The incidence of ESBL-E varied by region but showed an upward trend overall. Reinforcement of control measures for halting the spread of such MRO is crucial.

\section{Introduction}

In recent decades, the spread of multidrug-resistant organisms (MRO) has had a profound impact on healthcare facilities (HCF), combining a high mortality rate $(16 \%)$ and financial burden per patient (5,000-10,000 Euros per episode of bacteraemia due to extended-spectrum beta-lactamase-producing Enterobacteriaceae (ESBL-E) [1,2]. Multidrug resistance is a step towards a therapeutic dead-end and involves bacteria responsible for both healthcare-associated and community-acquired infections. MRO account for an important part of healthcare-associated infections, as shown in the national prevalence survey in France and in Europe in 2012 [3,4]. That year, the prevalence of ESBL-E in French hospitals was found to be $13.6 \%$ [5].

ESBL-E are challenging because of their pathogenicity, dissemination within hospitals and their potential reach into the community [6]. In addition, some extended-spectrum beta-lactamase-producing (ESBL) organisms such as Escherichia coli and Klebsiella pneumoniae can colonise a patient long after hospital discharge, especially in the digestive tract [7], which may facilitate their spread in the general population [8].

In France, until 2009, MRO control programmes focused on meticillin-resistant Staphylococcus aureus (MRSA), with a successful decrease in incidence (a significant reduction was seen from 2006) [9,10]. In contrast, however, incidence of ESBL-E increased during the same period, suggesting poor effectiveness of control measures [11-13]. ESBL-E surveillance has been carried out since 2002 by the French surveillance network for healthcare-associated infections (MRO RAISIN [9]) and has been a major focus of MRO surveillance programmes in France since 2010.

Here we present the 2009-13 ESBL-E surveillance data from the French MRO network, showing regional variation and temporal trends. 
Incidence of extended-spectrum beta-lactamase-producing Enterobacteriaceae infections by type of care ${ }^{\text {, healthcare }}$ facilities cohort, surveillance network for healthcare-associated infections database, France, 2009-13 (n = 32,201)

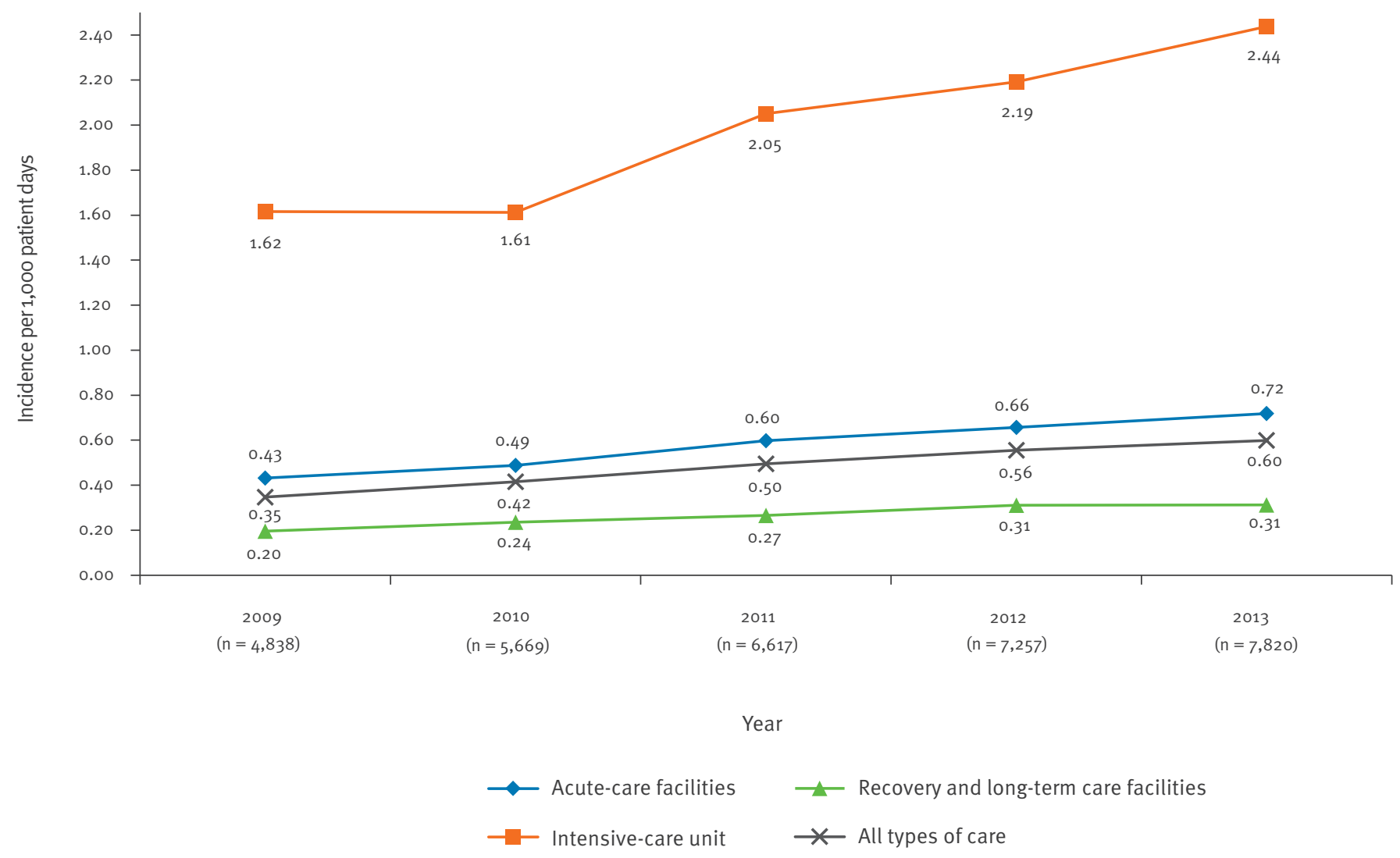

a Poisson's regression test, $\mathrm{p}=0.001$ for all types of care.

\section{Methods}

ESBL-E surveillance has been implemented by RAISIN using standardised methods described elsewhere [9]. Every year, all HCF with 24 hour patient-day hospitalisation in France are invited to participate in a threemonth survey on a voluntary basis. Online software was created by the Regional Coordinating Centre for Healthcare-Associated Infections Control in Paris to facilitate the hospitals' participation, with a userfriendly interface for entering data and controlling for errors (e.g. to check if the hospital unit from which the sample was taken is still in existence, if there is data variation of $\pm 20 \%$, when compared with administrative data of the previous year).

A case of ESBL-E infection was defined as a patient with at least one ESBL-E-positive diagnostic sample. ESBL-E strains were isolated from samples collected during the survey period for infection diagnosis purposes from patients who had been hospitalised for at least 24 hours (excluding dialysis and ambulatory care units, and the time for dialysis and ambulatory care). When multiple strains of the same species were isolated from the same patient, only the first strain was included in the surveillance network for healthcare-associated infections database, in order to avoid duplication of data.

Antibiotic susceptibility tests were performed according to the guidelines of the Committee for Antimicrobial Testing of the French Society of Microbiology [14,15]. Detection of ESBL production was based on synergy between third-generation cephalosporins and clavulanic acid [16]. Cases who were colonised or found to have community-acquired ESBL-E infections were not included in the study.

We analysed data collected in HCF that participated every year during the 2009-13 period (referred to as the HCF cohort), except for type of pathogen, for which we analysed data from all participating HCF. Incidence of ESBL-E infection was calculated per 1,000 in-hospital patient days (PD). Temporal linear trends were estimated using univariate Poisson regression analysis. Pooled incidence rates of ESBL-E infection were also represented on maps using 0.2 incidence gradient categories. We used SAS software release 9.2 (SAS, Cary, NC, United States) for all analyses. P values were significant at 0.05 . 
Incidence per 1,000 patients-days (PD) trends of Extended-spectrum beta-lactamase-producing (ESBLE) by region, healthcare facilities cohort, surveillance network for healthcare associated infections database, France, 2009-2013 (N ESBLE $=32,201)$
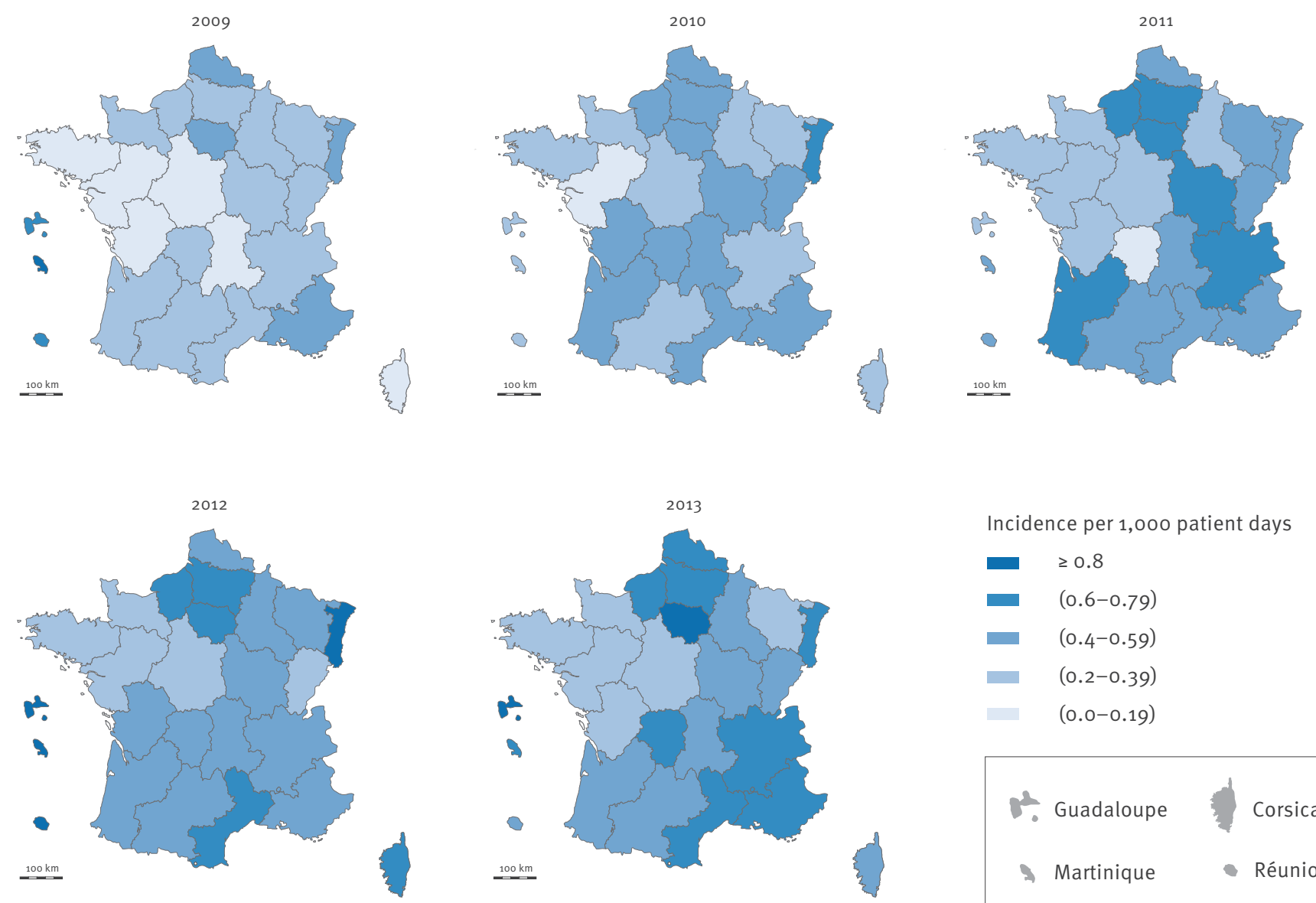

Incidence per 1,000 patient days

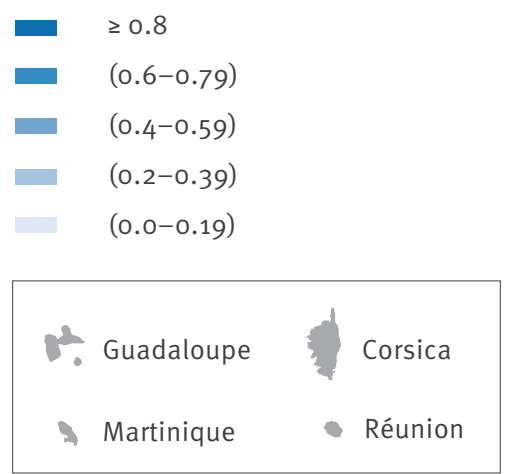

Map obtained from d-maps (http://www.d-maps.com/carte.php?num_car=18215\&lang=en).

\section{Results}

From 2009 to 2013, a cohort of 577 HCF participated each year in the survey, collecting 32,201 ESBL-E strains for diagnostic purposes. The incidence of ESBLE-positive samples increased overall from 0.35 to 0.60 per 1,000 PD ( $p<0.001$ ) from 2009 to 2013 respectively, corresponding to a $73 \%$ increase over the period. The incidence of ESBL-E infections varied according to type of care facility from 0.43 to 0.72 per 1,000 PD ( $p<0.001)$ in acute-care facilities, from 0.20 to 0.31 per 1,000 PD $(p<0.001)$ in recovery and long-term care facilities, with a higher incidence in intensive-care units (from 1.62 to 2.44 per 1,000 PD; $p<0.001$ ) (Figure 1). Incidence of ESBL-E bacteraemia increased from 0.03 to 0.05 per 1,000 PD (number of bacteraemia events: 425 in 2009, 704 in 2013), representing a $77 \%$ increase ( $p<0.001)$.

The incidence of ESBL-E infections increased nationwide but varied across regions (median $p$ value of Poisson regression test: 0.001 ; range: $0.001-0.37$ ). The highest incidences were observed in the eastern regions (+233\% increase; 0.18 to 0.59 per 1,000 PD in 2009 and 2013, respectively), in Guadeloupe, Martinique and Réunion (French overseas department and region): $+229 \%$ increase; from 0.57 to 0.92 per $1,000 \mathrm{PD})$ and in the northen regions, with the highest in 2013 being in the Paris area $(+71 \%$ increase; from 0.51 to 0.88 per $1,000 \mathrm{PD})$, whereas the lowest values were seen in western regions $(+28 \%$ increase; from 0.53 to 0.67 per 1,000 PD) (Figure 2)). In 2013, the incidence was greater than 0.35 per $1,000 \mathrm{PD}$ in all regions except some western regions. The incidence of ESBL-E infections significantly increased with the number of inhabitants $/ \mathrm{km}^{2}$ in each region $(p<0.001)$.

The number of participating HCF each year was respectively $929,933,974,1,181$ and 1,347 for respectively $5,946,6,992,8,475,10,778$ and 12,171 ESBL-E strains collected for three months per year between 2009 and 2013. Of the 44,362 infections, 26,195 (59\%) were due to E. coli, 8,844 (20\%) to K. pneumoniae and 5,006 


\section{FIGURE 3}

Extended-spectrum beta-lactamase-producing Enterobacteriaceae infections by pathogen, surveillance network for healthcare associated infections database, France, $2013(\mathrm{n}=12,234)$

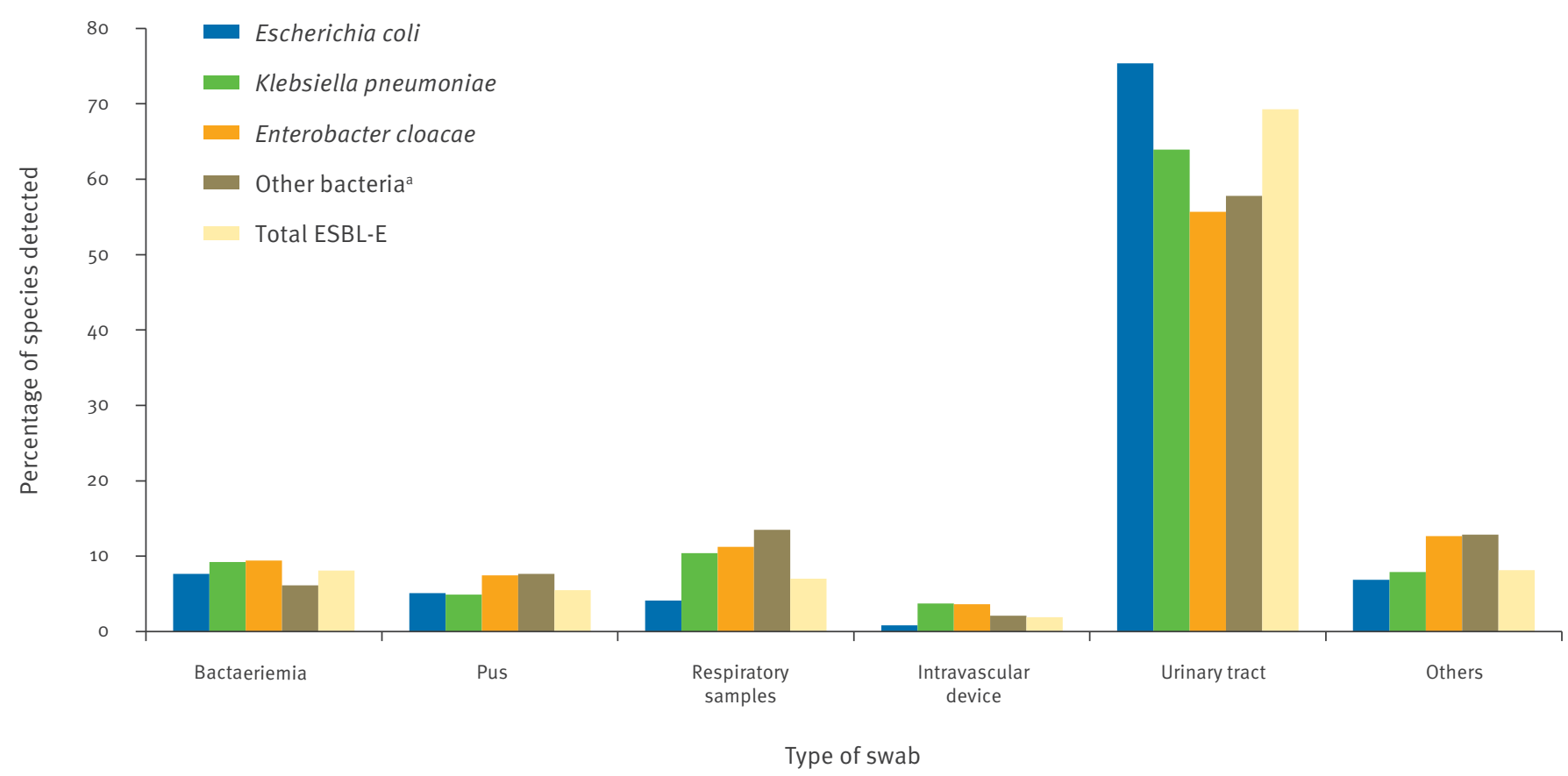

ESBLE: Extended-spectrum beta-lactamase-producing Enterobacteriaceae.

${ }^{a}$ Citrobacter spp, Enterobacter aerogenes, Klebsiella oxytoca, Proteus mirabilis and Serratia spp.

\section{FIGURE 4}

Incidence of extended-spectrum beta-lactamase-producing Enterobacteriaceae infections by species, surveillance network for healthcare associated infections database, France, 2009-13 $(n=44,362)$

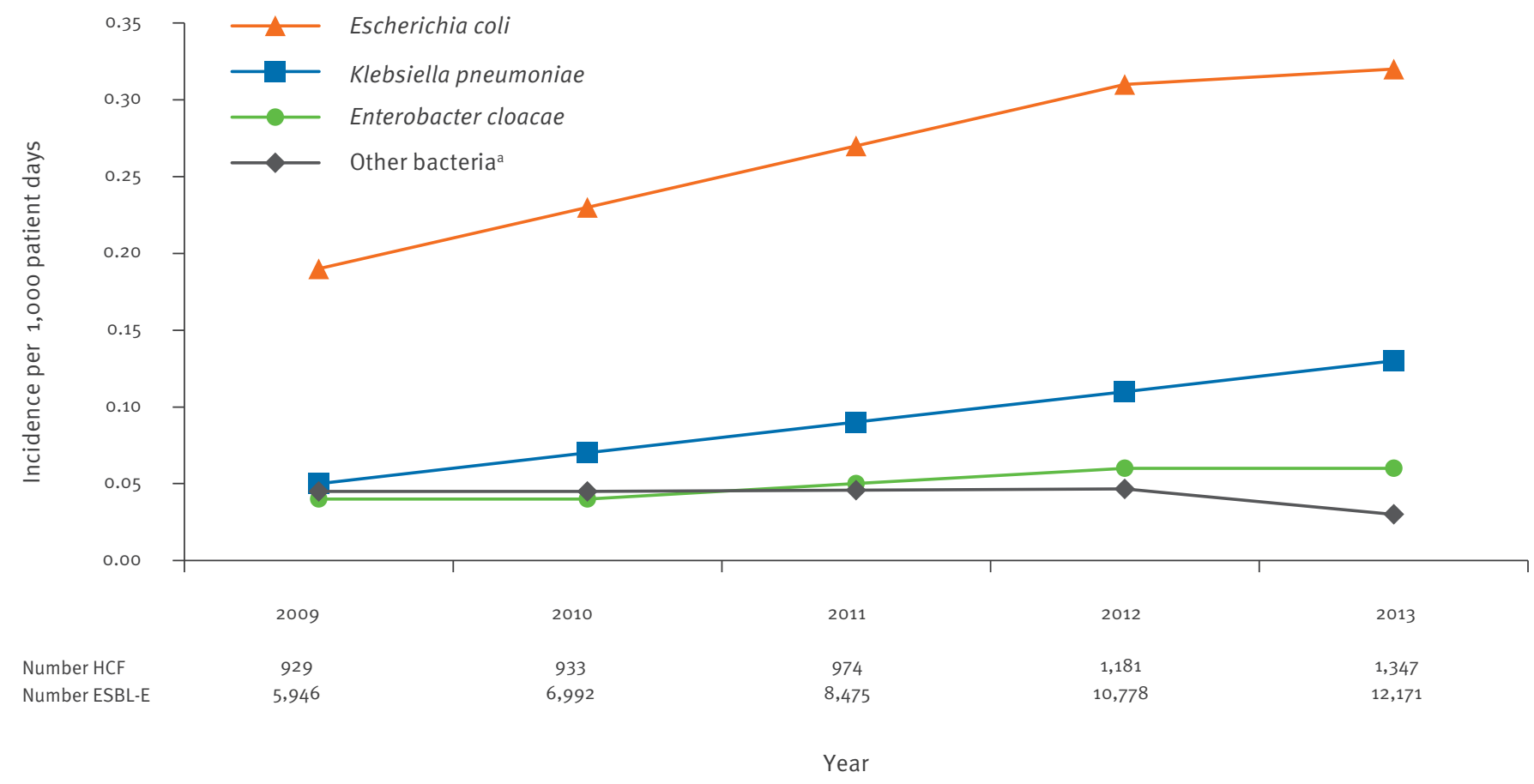

ESBLE: Extended-spectrum beta-lactamase-producing Enterobacteriaceae; HCF: healthcare facilities.

${ }^{a}$ Citrobacter spp, Enterobacter aerogenes, Klebsiella oxytoca, Proteus mirabilis and Serratia spp. 
(11\%) to Enterobacter cloacae. The most frequent infection in 2013 was urinary tract infection for all species, including mainly E. coli ( $75 \% ; 5,419 / 7,189$ E. coli). K. pneumoniae and $E$. cloacae were the most frequent pathogens isolated from patients with bacteraemia ( $\%$; 257/2,798 K. pneumoniae and 9\%; 126/1,345 E. cloacae) followed by E. coli $(8 \% ; 551 / 7,189$ E. coli). Details by type of swab and pathogens in 2013 are presented in Figure 3.

Since 2009, the proportion of ESBL-E urinary tract infections increased by $8 \%(3,826 / 5,946$ in 2009 to $8,478 / 12,234$ in 2013). The incidence of $E$. coli infections increased from 0.19 to 0.32 per 1,000 PD. The same upward trend was observed for $K$. pneumoniae (from 0.05 to 0.13 per 1,000 PD) and E. cloacae (from 0.04 to 0.06 per 1,000 PD) (Figure 4). Conversely, the incidence of other ESBLE species including $E$. aerogenes tended to decrease (from 0.03 to 0.01 per 1,000 PD for E. aerogenes and from 0.05 to 0.03 per 1,000 PD for the other bacteria including Citrobacter spp., Klebsiella oxytoca and Proteus mirabilis).

\section{Discussion}

Our study provides additional epidemiological data surveying ESBL-E in France and could help in promoting infection control policies against MRO in France. The important increase in the incidence of ESBL-E infections observed during the study period is worrisome.

In the mid-1980s, clusters of infections due to ESBL-K. pneumoniae were observed in French hospitals, predominantly in the Paris area [16]. After an effective campaign of infection control measures, including reinforcement of barrier precautions and early detection of carriers, incidence of ESBL-E infections began to decrease in 1993, suggesting that these pathogens could be brought under control. During the following 15 years, control efforts focused on the control of MRSA cross-infection and obtained significant curbing of incidence, with a regular decrease in the number of infections [10]. Surprisingly, however, this control programme had no impact on the incidence of ESBL-E infection, raising the question of whether extended or more appropriate control measures should be implemented, including management of excreta in healthcare settings. Indeed, the measures for controlling MRSA are different from those for Enterobacteriaceae, related to the route of transmission of those two types of pathogens [17], and could partly explain the lack of effectiveness of such programmes. The frequent transfer of ESBL-encoding genes among Enterobacteriacae species present in the flora of humans' and animals' digestive tract, combined with the faecal-oral route of transmission via the food chain $[18,19]$, could partly explain the rapid dissemination of ESBL-E in the community and as a result, in the healthcare setting $[13,20]$.

A slow increase in incidence of ESBL-E infections was seen in France from 2003 to 2006 (0.17 to 0.20 per 1,000 PD, respectively, for a $175 \mathrm{HCF}$ cohort) [9]. The substantial increase of ESBL-E infections observed since 2006, with an incidence increasing from 0.30 per 1,000 PD in 2007 to 0.35 per 1,000 PD in 2008 [9], has proved a challenge in France because of their wider spread in hospitals and their potential for favouring emergence of very highly resistant pathogens such as carbapenemase-producing Enterobacteriacae, due to antibiotic selection pressure [21]. The incidence of these infections continues to increase in France despite official guidelines and there is an urgent need to reinforce control measures based on early detection of cases, management of excreta and improvement of antibiotic use, including in extra-hospital settings such as nursing homes and home-based hospital care.

A similar increase in the number of ESBL-E infections, particularly E. coli and K. pneumoniae, was observed in other European countries in 2005-06 [22]. Data from the European Antimicrobial Resistance Surveillance Network (EARS-Net) showed an average of a $20-25 \%$ increase of third-generation cephalosporin resistance in every country in the network between 2007 and 2010, with between $85 \%$ and $100 \%$ ESBL-positive isolates among third-generation cephalosporin-resistant strains [23,24]. However, the incidence of ESBL-E infections seen in France in 2013 was one of the highest in Europe [22].

The increased of number of ESBL-E infections in France is unlikely to be explained by an increase in sample collection over the years due to better awareness or systematic screening by the participating centres, as only samples collected for diagnostic purposes were included. However, it is possible that clinicians' awareness increased during the study period. In addition, clusters of ESBL-E infection were not observed more frequently through the French mandatory reporting system (e-SIN) during the study period [25] so local outbreaks would not explain this increase in ESBL-E infections.

In France, the incidence of ESBL-E. coli and -K. pneumoniae infections increased steadily from 2006 to 2013, whereas the incidence of infections due to ESBL-E. aerogenes and other species decreased steadily (the percentage distribution of the main species of ESBL-E in 2002-10 can be found in [9]). During this time, $E$. coli was the major pathogen among ESBL-E, especially in intensive-care units, where the incidence was double that seen in all other settings. Indeed, ESBL-E. coli poses a potential threat of high burden to HCF and related facilities (such as nursing homes and homebased hospital care) $[2,26]$. This pathogen is the one of the most frequently isolated in both community and hospital-acquired urinary tract infections, and could be the cause of severe or fatal outcomes associated with bacteraemia, which has been shown to be increasing in France [27], with a significant increase in incidence of ESBL-E bacteraemia between 2009 and 2013 from 0.021 to 0.044 per 1,000 PD, respectively. The burden of ESBL-E bacteraemia, including $E$. coli, was reported 
in studies in several European counties (Austria, Belgium, Croatia, England, Germany, Greece, Ireland, Italy, Latvia, Malta, Romania, Scotland and Slovenia) in 2008 [26] and, more specifically, in Switzerland in 2009 to be five to seven excess days in hospital per hospital stay, at a cost of about 8,000 Euros per bacteraemia episode [2].

Our study showed a statistically significant geographical variation in France, with incidence being two to fourfold higher in some regions. These results are based on actual estimates of incidence rates, not the proportion of ESBL among Enterobacteriacae, which is a strength of this study. Few other studies, notably in the United States in 2012 and Portugal in 2010, have compared the geographical distribution of the incidence of ESBL-E infection, showing substantial differences according to the species, with predominance of E. coli and K. pneumoniae $[28,29]$, as in our study.

The incidence of ESBL-E infections was particularly high in Paris (northern region), Marseille and Lyon (south-east region), where there are the largest university hospitals in the country. Because of their proximity to national borders and airports, for example, these tertiary hospitals receive many foreigners and also repatriated French individuals with severe diseases or multiple wounds who had previous carriage of MRO [30]. Additionally, southern France is close to countries that are highly endemic for MRO such as Italy, Spain, Greece and northern African countries [19,31].

The high incidence rate observed in Guadeloupe, Martinique and Réunion (French overseas department and region) should be interpreted with caution because of the small size of the study population and the small number of HCF participating in the surveillance (two for each Island). Therefore, the incidence of ESBL-E infections could not be precisely estimated in these regions, generating potential classification bias. Regional incidence variations could be influenced by the relative burden placed on public HCF with a higher incidence. Indeed, public HCF are generally larger and receive high-risk patients (those who are elderly, in intensive care and those transferred from other HCF) more frequently than private HCF do. Additional epidemiological information is needed to better explore the factors influencing these trends.

National Early Warning, Investigation and Surveillance of Healthcare-Associated Infections Network (RAISIN)/ multidrug resistance study group

Odile Bajolet, Xavier Bertrand, Hervé Blanchard, Emmanuelle Caillat-Vallet, Bruno Coignard, Lory Dugravot, Catherine Dumartin, Mathieu Eveillard, Thierry Fosse, Nadine Garreau, Sophie Glorion, Jessica Lacoste, Olivier Hoff, Nicole Marty, Pierre Parneix, Emmanuelle Reyreaud, Anne Savey, Hélène Sénéchal, Loïc Simon, Elisabeth Sousa, David Trystram, Sophie Vaux.
Acknowledgements

The MRO Surveillance National Steering Group also includes Bajolet $\mathrm{O}$, Bertrand X, Blanchard H, Caillat-Vallet E, Coignard B, Dugravot L, Dumartin C, Eveillard M, Fosse T, Garreau N, Glorion S, Lacoste J, Hoff O, Marty N, Parneix P, Reyreaud E, Savey A, Sénéchal H, Simon L, Sousa E, Trystram D, Vaux S and the participating healthcare facilities.

\section{Conflict of interest}

None declared.

\section{Authors' contributions}

All authors have contributed directly to the intellectual content of the paper and have agreed to have their name listed as an author on the final, revised version. Their own substantive contribution to the paper is as follows: Isabelle Arnaud developed the concept of the manuscript, managed the national database, analysed the data and wrote the first draft of the manuscript. Sylvie Maugat contributed to interpret the results critically and revised the article to ensure important intellectual content. Vincent Jarlier provided critical revision of the article for important content. Pascal Astagneau provided epidemiological expertise, and also contributed to final revision.

\section{References}

1. ColodnerR, RockW, ChazanB, KellerN, GuyN, SakranW, et al. Risk factors for the development of extended-spectrum betalactamase-producing bacteria in nonhospitalized patients. Eur J Clin Microbiol Infect Dis. 2004;23(3):163-7. DOI: 10.1007/ S10096-003-1084-2 PMID: 14986159

2. StewardsonA, FankhauserC, De AngelisG, RohnerP, SafranE, SchrenzelJ, et al. Burden of bloodstream infection caused by extended-spectrum $\beta$-lactamase-producing enterobacteriaceae determined using multistate modeling at a Swiss University Hospital and a nationwide predictive model. Infect Control Hosp Epidemiol. 2013;34(2):133-43. DOI: 10.1086/669086 PMID: 23295559

3. WegnerC, HübnerNO, GleichS, ThalmaierU, KrügerCM, KramerA. One-day point prevalence of emerging bacterial pathogens in a nationwide sample of 62 German hospitals in 2012 and comparison with the results of the one-day point prevalence of 2010.GMS Hyg Infect Control. 2013;8(1):Doc12. PMID: 23967398

4. National Contact Points for the ECDC pilot point prevalence survey,ZarbP, CoignardB, GriskevicieneJ, MullerA, VankerckhovenV, WeistK, et al. . The European Centre for Disease Prevention and Control (ECDC) pilot point prevalence survey of healthcare-associated infections and antimicrobial use.Euro Surveill. 2012;17(46):20316.PMID: 23171822

5. Réseau d'alerte, d'investigation et de surveillance des infections nosocomiales (RAISIN). Enquête nationale de prévalence des infections nosocomiales et des traitements anti-infectieux en établissements de santé, France, maijuin 2012. Résultats. [2012 national prevalence survey of nosocomial infections and anti-infection treatments in healthcare facilities. May-June 2012. Results]. Saint-Maurice: Institut de veille sanitaire; 2013. [Accessed 2 Sep 2015]. French. Available from: http://www.invs.sante.fr/Publicationset-outils/Rapports-et-syntheses/Maladies-infectieuses/2013/ Enquete-nationale-de-prevalence-des-infectionsnosocomiales-et-des-traitements-anti-infectieux-enetablissements-de-sante-France-mai-juin-2012

6. GoulenokT, FerroniA, BilleE, LécuyerH, Join-LambertO, DescampsP, et al. Risk factors for developing ESBL E. coli: can clinicians predict infection in patients with prior colonization? Hosp Infect. 2013;84(4):294-9. DOI: 10.1016/j.jhin.2013.04.018 PMID: 23846237

7. BirgandG, Armand-LefevreL, Loloml, RuppeE, AndremontA, LucetJC. Duration of colonization by extended-spectrum $\beta$-lactamase-producing Enterobacteriaceae after hospital discharge.Am J Infect Control. 2013;41(5):443-7. DOI: 10.1016/j. ajic.2012.05.015 PMID: 22998785

8. ValverdeA, CoqueTM, Sánchez-MorenoMP, RollánA, BaqueroF, CantónR. Dramatic increase in prevalence of fecal 
carriage of extended-spectrum beta-lactamase-producing Enterobacteriaceae during nonoutbreak situations in Spain.J Clin Microbiol. 2004;42(10):4769-75. DOI: 10.1128/ JCM.42.10.4769-4775.2004 PMID: 15472339

9. MDRB Surveillance National Steering Group (BMR-

Raisin), CarbonneA, Arnaudl, MaugatS, MartyN, DumartinC, BertrandX, et al. . National multidrug-resistant bacteria (MDRB) surveillance in France through the RAISIN network: a 9 year experience.J Antimicrob Chemother. 2013;68(4):954-9. DOI: 10.1093/jac/dks464 PMID: 23194721

10. Collégiale de Bactériologie-Virologie-Hygiène des Hôpitaux Universitaires de l'lle de France,JarlierV, TrystramD, BrunBuissonC, FournierS, CarbonneA, MartyL, et al. . Curbing methicillin-resistant Staphylococcus aureus in 38 French hospitals through a 15-year institutional control program. Arch Intern Med. 2010;170(6):552-9. DOI: 10.1001/ archinternmed.2010.32 PMID: 20308642

11. Toronto ESBL Working Group,LoweCF, KatzK, McGeerAJ, MullerMP. Efficacy of admission screening for extended spectrum beta-lactamase producing Enterobacteriaceae.PLoS ONE. 2013;8(4):e62678. DOI: 10.1371/journal.pone.0062678 PMID: 23638132

12. GiboldL, RobinF, TanRN, DelmasJ, BonnetR. Four-year epidemiological study of extended-spectrum $\beta$-lactamaseproducing Enterobacteriaceae in a French teaching hospital. Clin Microbiol Infect. 2014;20(1):020-6. DOI: 10.1111/14690691.12321 PMID: 23927626

13. KassakianSZ, MermelLA. Changing epidemiology of infections due to extended spectrum beta-lactamase producing bacteria.Antimicrob Resist Infect Control. 2014;3(1):9. DOI: 10.1186/2047-2994-3-9 PMID: 24666610

14. SoussyCJ, CarretG, CavalloJD, ChardonH, ChidiacC, ChoutetP, et al. [Antibiogram Committee of the French Microbiology Society. Report 2000-2001]. Pathol Biol (Paris). 2000;48(9):832-71.PMID: 11141919

15. French Society of Microbiology (SFM). Comité de l'antibiogramme de la Société Française de Microbiologie. Recommandation 2015. [Committee for Antimicrobial Testing of the French Society of Microbiology. 2015 recommendations]. Paris: SFM. [Accessed 2 Sep 2015]. French. Available from: http://www.sfm-microbiologie.org/UserFiles/files/casfm/ CASFMV 2 220715.pdf

16. JarlierV, NicolasMH, FournierG, PhilipponA. Extended broad-spectrum beta-lactamases conferring transferable resistance to newer beta-lactam agents in Enterobacteriaceae: hospital prevalence and susceptibility patterns. Rev Infect Dis. 1988;10(4):867-78. DOI: 10.1093/clinids/10.4.867 PMID: 3263690

17. Lin M, Weinstein RA, Hayden MK. Multiply drug-resistant pathogens: epidemiology and control. In: Jarvis WR, editor. Bennett \& Brachman's hospital infections. 5 th ed. Philadelphia: Lippincott Williams \& Wilkins; 2007. p. 193-222.

18. ReichF, AtanassovaV, KleinG. Extended-spectrum $\beta$-lactamaseand AmpC-producing enterobacteria in healthy broiler chickens, Germany.Emerg Infect Dis. 2013;19(8):1253-9. DOI: 10.3201/eid1908.120879 PMID: 23876576

19. GeserN, StephanR, HächlerH. Occurrence and characteristics of extended-spectrum $\beta$-lactamase (ESBL) producing Enterobacteriaceae in food producing animals, minced meat and raw milk.BMC Vet Res. 2012;8(1):21. DOI: 10.1186/17466148-8-21 PMID: 22397509

20. van HoekAH, SchoulsL, van SantenMG, FlorijnA, de GreeffSC, van DuijkerenE. Molecular characteristics of extendedspectrum cephalosporin-resistant Enterobacteriaceae from humans in the community.PLoS ONE. 2015;10(6):e0129085. DOI: 10.1371/journal.pone.0129085 PMID: 26029910

21. RAISIN and Expert Laboratories Groups,VauxS, CarbonneA, ThioletJM, JarlierV, CoignardB. Emergence of carbapenemaseproducing Enterobacteriaceae in France, 2004 to 2011.Euro Surveill. 2011;16(22):19880.PMID: 21663708

22. European Centre for Disease Prevention and Control (ECDC). Antimicrobial resistance interactive database (EARS-Net). Stockholm: ECDC. [Accessed 2 Sep 2015]. Available from: http://ecdc.europa.eu/en/healthtopics/antimicrobial resistance/database/Pages/database.aspx\#sthash.goMyoHLp. dpuf

23. European Centre for Disease Prevention and Control (ECDC). Antimicrobial resistance surveillance in Europe 2012. Annual Report of the European Antimicrobial Resistance Surveillance Network (EARS-Net). Stockholm: ECDC; 2013. Available from: http://ecdc.europa.eu/en/publications/Publications/ antimicrobial-resistance-surveillance-europe-2012.pdf

24. JonesRN, FlontaM, GurlerN, CepparuloM, MendesRE, CastanheiraM. Resistance surveillance program report for selected European nations (2011).Diagn Microbiol Infect Dis.
2014;78(4):429-36. DOI: 10.1016/j.diagmicrobio.2013.10.008 PMID: 24440509

25. Institut de Veille Sanitaire (InVS). Signalement externe des infections nosocomiales. E-SIN. [External reporting of nosocomial infections. E-SIN]. Saint Maurice: InVS. [Accessed 2 Sep 2015]. Available from: http://www.invs. sante.fr/Dossiers-thematiques/Maladies-infectieuses/ Infections-associees-aux-soins/Signalement-et-alertes/ Signalement-externe-des-infections-nosocomiales

26. de KrakerME, WolkewitzM, DaveyPG, KollerW, Berger], Nagler), et al. Burden of antimicrobial resistance in European hospitals: excess mortality and length of hospital stay associated with bloodstream infections due to Escherichia coli resistant to third-generation cephalosporins. J Antimicrob Chemother. 2011;66(2):398-407. DOI: $10.1093 / j a c / d k q 412$ PMID: 21106563

27. Institut de Veille Sanitaire (InVS). Surveillance des bactéries multiresistantes dans les établissements de santé français. Réseau BMR-Raisin - Données 2012, France 01-01-2014. [Surveillance of multidrug-resistant bacteria in Ffrench health care facilities. MRO RAISIN network. 2012 results. France, 01-01-2014]. Saint Maurice: InVS. [Accessed 3 Sep 2015]. French. Available from: http://www.invs.sante.fr/pmb/ invs/\%28id\%29/PMB_12101

28. CastanheiraM, FarrellSE, KrauseKM, JonesRN, SaderHS. Contemporary diversity of $\beta$-lactamases among Enterobacteriaceae in the nine U.S. census regions and ceftazidime-avibactam activity tested against isolates producing the most prevalent $\beta$-lactamase groups.Antimicrob Agents Chemother. 2014;58(2):833-8. DOI: 10.1128/AAC.0189613 PMID: 24247134

29. Fernandes R, Amador P, Oliveira C, Prudêncio C. Molecular Characterization of ESBL-Producing Enterobacteriaceae in Northern Portugal. ScientificWorldJournal. 2014;2014:782897. doi: $10.1155 / 2014 / 782897$

30. Institut de Veille Sanitaire (InVS). Episodes impliquant des entérobactéries productrices de carbapénèmases en France. Situation épidémiologique du 4 mars 2015. [Episodes involving carbapenemases producing enterobacteria in France. Epidemiological situation of March 4, 2015]. Saint Maurice: InVS. [Accessed 2 Sep 2015]. Available from: http://www.invs.sante.fr/Dossiers-thematiques/ Maladies-infectieuses/Infections-associees-aux-soins/ Surveillance-des-infections-associees-aux-soins-IAS/ Enterobacteries-productrices-de-carbapenemases-EPC/ Episodes-impliquant-des-enterobacteries-productrices-decarbapenemases-en-France.-Situation-epidemiologique-du-4mars-2015

31. Djahmi N, Dunyach-Remy C, Pantel A, Dekhil M, Sotto A, Lavigne JP. Epidemiology of carbapenemase-producing Enterobacteriaceae and Acinetobacter baumannii in Mediterranean countries. Biomed Res Int. 2014;2014:305784. 